\title{
Advances in the management of cardiovascular disease during pregnancy
}

\author{
Benjamin Viaris de Lesegno*,1 \& Catherine Nelson-Piercy ${ }^{1}$ \\ 'Guy's and St Thomas' NHS Foundation Trust, St Thomas' Hospital, London SE1 7EH, UK \\ *Author for correspondence: benjamindeviaris@gmail.com
}

\begin{abstract}
"It is important to start therapy prior to pregnancy in order to evaluate the response, optimize the treatment and hope to make the pulmonary vasculature less reactive to changes that will occur during pregnancy."
\end{abstract}

First draft submitted: 8 February 2018; Accepted for publication: 25 May 2018; Published online: 25 June 2018

Keywords: anticoagulation • cardiac disease • mitral stenosis • pregnancy • pulmonary hypertension

The United Kingdom Confidential Enquiries into Maternal Deaths is the longest running audit on maternal deaths in the world. It investigates and highlights preventable causes of maternal mortality [1]. Cardiovascular diseases remained the leading cause of maternal deaths in the 2017 report. This report also identified that there was no significant decrease in the mortality rate from cardiovascular diseases between 2010-2012 ( $\mathrm{n}=54)$ and 2013-2015 $(\mathrm{n}=54)$. The predominant take-home message from this report is the importance of providing women with cardiovascular disease the opportunity to benefit from a joint obstetric/cardiology clinic, or from the care of a cardiologist with experience in the care of pregnant women. But there remains a great variation in practice and expertise in the care of these patients.

This journal published our review article on cardiac disease in pregnancy in 2014 [2] and this current article aims to highlight and summarize the advances in the knowledge of care of these patients. We intend to update the reader on important literature published since our last review.

\section{Pulmonary hypertension}

Pulmonary arterial hypertension $(\mathrm{PAH})$ can be associated with connective tissue disease, be secondary to lung disease, to chronic hypoxia, to chronic thromboembolism or to a structural heart disease. It can also be idiopathic. Right ventricular failure and premature death are its most severe complications. Usually a maternal mortality of $25 \%$ in pregnancy is quoted and $7 \%$ in case of early termination of pregnancy [3,4]. More recent studies which postdate the use of targeted therapies for pulmonary hypertension quote lower (10\%) mortality rates. However, the current joint guideline from the European Society of Cardiology and European Respiratory Society continues to recommend the avoidance of pregnancy for women with PAH [5].

The safety of medical therapies for PAH in pregnancy is difficult to assess and there is a lack of literature on this subject. Outside pregnancy, the standard therapy is a combination of an endothelin receptor antagonist (ERA) and a phosphodiesterase type 5 inhibitor (PDE 5i), in particular ambrisentan and tadalafil [6]. But ERAs have been shown to be teratogenic in rats and should not be used in the first trimester. On the other hand tadalafil is safe in pregnancy. There is no evidence from randomized controlled trials, but the use of pulmonary vasodilators such as oral/intravenous/nebulized prostacyclin, sildenafil or bosentan (an ERA) with successful outcomes have been described. The outcome of pregnancy in PAH patients seems to improve when PAH is well controlled, especially in responders to calcium channel blockers. It is important to start therapy prior to pregnancy in order to evaluate the response, optimize the treatment and hope to make the pulmonary vasculature less reactive to changes that will occur during pregnancy. It is advised to continue during pregnancy the therapy that was effective before pregnancy, unless the patient is receiving an ERA [7]. If the patient is receiving a monotherapy with ERA, she should switch to a monotherapy with PDE 5i, and if receiving combination therapy with ERA and PDE 5i, she should switch 
to a combination of PDE 5i and iloprost or withdraw the ERA in the first trimester and restart after 12 weeks gestation [7].

\section{Valvular heart disease \& valve prostheses}

The site of the valvular heart disease and its severity are the key factors in managing pathology in pregnancy. All patients with valvular disease, with or without prostheses should undergo clinical evaluation, echocardiography and counselling prior to pregnancy. It is recommended that women with severe aortic stenosis or mitral stenosis undergo surgical or percutaneous corrective procedures before pregnancy. Stenotic valves, particularly left-sided, are at a higher risk of maternal compromise than regurgitant valves, as the physiological vasodilatation and tachycardia of pregnancy will be less well tolerated. Antibiotic prophylaxis against infective endocarditis during obstetric procedures or childbirth is not recommended by the current NICE guidelines [8]. Method of delivery is determined by a multidisciplinary team and depends on numerous factors: presence of heart failure or PAH, functional capacity and exercise tolerance, need for therapeutic anticoagulation and past obstetric history. Elective caesarean section is not usually recommended but may be indicated if there is a risk of decompensation during vaginal delivery or to plan the presence of a multidisciplinary team in the highest risk women.

The main issue in patients with mechanical valves is the need for therapeutic anticoagulation in pregnancy. These patients should be referred to specialist clinics as early as possible and ideally have a plan made prior to any pregnancy. The most widely used oral anticoagulant is warfarin. All the novel anticoagulants (Apixaban, Edoxaban, Dabigatran and Rivaroxaban) should not be used in pregnancy. The risk of valve thrombosis and, therefore, associated maternal morbidity is lower with oral anticoagulants used throughout pregnancy, when compared with substitution with low molecular weight heparin (LMWH) in the first trimester [9]. Warfarin is associated with warfarin embryopathy causing nasal hypoplasia and bone defects, and with increased risk of miscarriage, stillbirth and fetal intracerebral hemorrhage [10]. The greatest risk of warfarin embryopathy is between 5 and 12 weeks' gestation. In women with whom the decision has been taken to switch from warfarin to LMWH in the first trimester, this should be achieved before 5 weeks of gestation. These risks are dose dependent [11]. Using a low dose of warfarin (less than $5 \mathrm{mg} /$ day) may diminish this risk but predispose to valve thrombosis if this is not adequate to achieve a therapeutic international normalized ratio. Therefore continuation of warfarin during the first trimester is reasonable for a pregnant patient with a mechanical valve if the dose to achieve target international normalized ratio is less than $5 \mathrm{mg} /$ day and after discussion of all risks and benefits with the patient. Nonetheless, a small risk remains with a warfarin dose of less than $5 \mathrm{mg} /$ day.

The risk of embolic event or valve thrombosis increases with LMWHs compared with warfarin [11,12]. During the course of the pregnancy, the dose requirement increases because of accelerated renal clearance and increased volume of distribution. Anti-Xa levels therefore should be monitored. Target anti-Xa 4-6 h post dose is $0.8-1.2$ when using LMWH. If LMWH is used, low-dose aspirin should be coprescribed.

Unfractionated heparin and LMWH do not cross the placenta and have no harmful effect on the fetus but there is a higher rate of thromboembolic complications with subcutaneous unfractionated heparin. Therefore, it is not recommended during pregnancy in the 2014 AHA/ACC guideline [13].

Planned delivery is preferred and its modality discussed in a multidisciplinary setting, in order to safely transition and manage anticoagulation. If warfarin is used, it should be stopped prior to delivery to allow the fetus to clear the drug (usually 36 weeks). Timing of the transition from warfarin to LMWH must be adapted for patients at high risk of preterm labor. Vaginal delivery is the preferred mode of delivery and cesarean section should be reserved for obstetric indications. Delivery (vaginal or by cesarean section) is not an indication for antibiotic prophylaxis. In the absence of bleeding complications, the anticoagulation should be resumed 4-6 h after the delivery with unfractionated heparin or LMWH and then conversion to warfarin at least 5 days postpartum. Warfarin is compatible with breastfeeding but direct oral anticoagulants should be avoided or the woman is advised not to breastfeed.

\section{Ischemic heart disease \& myocardial infarction}

Pregnancy increases the risk of myocardial infarction. With the increase in prevalence of cardiovascular risk factors such as hypertension, obesity, diabetes mellitus and dyslipidemia in the maternal population, it is likely that the rate of myocardial infarction will increase in future years. In pregnancy, the most common form of myocardial infarction is secondary to coronary atherosclerosis, but spontaneous coronary artery dissection and in situ thrombosis and embolism are relatively more common in pregnancy than in the nonpregnant [14]. Diagnosis in the pregnant 
population is the same as outside of pregnancy with dynamic ECG changes and troponin rise. It is important to keep in mind that a normal ECG and/or a negative troponin does not exclude the diagnosis of an acute coronary syndrome, as specified in the MBBRACE-UK report [1]. Management of acute coronary syndromes should not be delayed and the patient should be offered immediate percutaneous coronary intervention. Coronary angioplasty, thrombolysis and stenting have all been successfully reported in pregnancy [14-16]. In view of the reduced duration of dual antiplatelet therapy, bare-metal stents are preferable. In case of a cardiac arrest, early recourse to perimortem caesarean section is essential and an important part of the resuscitation process. Secondary prevention and management of risk factors is primarily lifestyle modification. Statins should be discontinued ideally prior to pregnancy. In pregnancy, it is safe to use antiplatelet agents but clopidogrel needs to be discontinued 1-2 weeks before delivery in order to diminish the risk of bleeding during regional analgesia/anesthesia. In patients with a history of myocardial infarction, further asymptomatic ischemia may occur, therefore treadmill exercise tolerance testing and myocardial perfusion imaging are recommended prior to pregnancy.

\section{Conclusion}

The latest MBRRACE report does not seem to reflect any improvement in the maternal mortality related to cardiac diseases [1]. In the last four years, more understanding of the care of cardiac patients has been achieved, especially patients with pulmonary hypertension, mechanical valves and ischemic heart disease [7,13,14]. We hope this article helps summarize this new knowledge and highlight to our readers the best care for our patients.

\section{Acknowledgements}

The authors would like to thank E Roche-Kelly who was one of the authors on the previous article on managing cardiovascular disease during pregnancy published in 2014.

\section{Financial \& competing interests disclosure}

The authors have no relevant affiliations or financial involvement with any organization or entity with a financial interest in or financial conflict with the subject matter or materials discussed in the manuscript. This includes employment, consultancies, honoraria, stock ownership or options, expert testimony, grants or patents received or pending, or royalties. No writing assistance was utilized in the production of this manuscript.

\section{References}

1 Knight M, Nair M, Tuffnell D, Shakespeare J, Kenyon S, Kurinczuk JJ (Eds); on behalf of MBRRACE-UK. Saving Lives, Improving Mothers' Care - Lessons learned to Inform Maternity Care from the UK and Ireland Confidential Enquiries into Maternal Deaths and Morbidity 2013-15. National Perinatal Epidemiology Unit, University of Oxford, Oxford, UK (2017).

2 Roche-Kelly E, Nelson-Piercy C. Managing cardiovascular disease during pregnancy: best practice to optimize outcomes. Future Cardiol. 10(3), 421-433 (2014).

3 Bedard E, Dimopoulos K, Gatzoulis MA. Has there been any progress made on pregnancy outcomes among women with pulmonary arterial hypertension? Eur. Heart J. 30, 256-265 (2009).

4 Weiss BM, Zemp L, Seifert B, Hess OM. Outcome of pulmonary vascular disease in pregnancy: a systematic overview from 1978 through 1996. J. Am. Coll. Cardiol. 31(7), 1650-1657 (1998).

5 Galiè N, Humbert M, Vachiery JL et al. 2015 ESC/ERS Guidelines for the diagnosis and treatment of pulmonary hypertension. Eur. Heart J. 37(1), 67-119 (2016).

6 Galiè N, Barbera JA, Frost A et al. Initial use of ambrisentan plus tadalafil in pulmonary arterial hypertension. N. Engl. J. Med. 379(9), 834-844 (2015).

7 Kiely DG, Condliffe R, Wilson VJ, Gandhi SV, Elliot CA. Pregnancy and pulmonary hypertension: a practical approach to management. Obstet. Med. 6(4), 144-154 (2013).

8 Centre for Clinical Practice at NICE (UK). Prophylaxis against infective endocarditis: antimicrobial prophylaxis against infective endocarditis in adults and children undergoing interventional procedures. NICE Clinical Guidelines 64 (2008). www.ncbi.nlm.nih.gov/pubmedhealth/PMH0009942/

9 Abildgaard U, Sandset PM, Hammerstrom J, Gjestvang FT, Tveit A. Management of pregnant women with mechanical heart valve prosthesis: thromboprophylaxis with low molecular weight heparin. Thromb. Res. 124(3), 262-267 (2009).

10 van Hagen IM, Roos-Hesselink JW, Ruys TP et al. with ROPAC investigators and the EURObservational Research Programme (EORP) team. Pregnancy in women with a mechanical heart valve: data of the European Society of Cardiology Registry of Pregnancy and Cardiac Disease (ROPAC). Circulation 132(2), 132-142 (2015). 
11 Lawley CM, Lain SJ, Algert CS, Ford JB, Figtree GA, Roberts CL. Prosthetic heart valves in pregnancy, outcomes for women and their babies: a systematic review and meta-analysis. BJOG 122(11), 1446-1455 (2015).

12 D'Souza R, Ostro J, Shah PS et al. Anticoagulation for pregnant women with mechanical heart valves: a systematic review and meta-analysis. Eur. Heart J. 38(19), 1509-1516 (2017).

13 Nishimura RA, Otto CM, Bonow RO et al. 2014 AHA/ACC guideline for the management of patients with valvular heart disease: a report of the American College of Cardiology/American Heart Association Task Force on Practice Guidelines. J. Am. Coll. Cardiol. 63(22), e57-e185 (2014).

14 Bush N, Nelson-Piercy C, Spark P et al. Myocardial infarction in pregnancy and postpartum in the UK. Eur. J. Prev. Cardiol. 20(1), 12-20 (2013).

15 Ladner HE, Danielsen B, Gilbert WM. Acute myocardial infarction in pregnancy and the puerperium: a population-based study. Obstet. Gynecol. 105(3), 480-484 (2005).

16 James AH, Jamison MG, Biswas MS, Brancazio LR, Swamy GK, Myers ER. Acute myocardial infarction in pregnancy: a United States population-based study. Circulation 113(12), 1564-1571 (2006). 